\title{
Research on the Dark Stripes Extraction Algorithm for Measuring Diameter with Diffraction
}

\author{
Quan Liu, Fenglin Wei \\ Changchun University of Science and Technology, Changchun, China \\ Email: liuquancust@126.com, 492982227@qq.com
}

Received 2013

\begin{abstract}
The optical diffraction is a major means of minute diameter measurements. Since the light intensity of the diffraction fringe, mainly distributed in the central bright stripes, and that will lead to a relatively low SNR for the signal of the senior stripes. The traditional dark stripes extraction algorithm affected greatly by the noise. So this paper presents an algorithm that could effectively eliminate the gross errorcaused by the noise. Than by curve fitting the local signal of the diffraction pattern with the least square principle, the system could achieve a high measurement precision.
\end{abstract}

Keywords: Diffraction; Diameter Measurement; Dark Stripes Extract; Least Squares Fitting

\section{Introduction}

Diffraction measurement method has attracted wide attention since its birth. According to the optical principle, when measured components has a smaller diameter, the corresponding diffraction phenomenon would be more obvious, so the diffraction measurement method is more suitable to the small diameter measurement ${ }^{[1-2]}$. During the diffraction measurement process, one single measurement needs to carry on a location measurement for the dark stripes in order to improve the measurement precision of position measurement. However as a result of diffraction, the main power of the diffraction pattern located in the central bright fringe, which lead to the highlevel fringes has a low SNR, which will cause a decline in measurement accuracy for the dark stripes location. The traditional dark stripes location extraction algorithm is unable to eliminate the gross error, which caused by the system noise, effectively. This paper presents an algorithm that could effectively eliminate the gross error caused by the noise. By curve fitting the local signal of the diffraction pattern with the least square principle, the system could achieve a high measurement precision.

\section{Diffraction Measurement Principle}

According to the Barbie pinch complementary principle, we know that the measurement for distribution of the diffracted light intensity of the measured filaments, can be equivalent to the measurement for the diffraction pattern of a slits which has a equal width, and we can get the formula:

$$
I=I_{0}\left(\frac{\sin ^{2} \beta}{\beta^{2}}\right)
$$

In which:

$$
\beta=\left(\frac{\pi b}{\lambda}\right) \sin \theta
$$

where: $\theta$ is the diffraction angle, $I_{0}$ is the Light intensity when $\theta=0$. The corresponding light intensity distribution, that cause by slit diffraction, mainly concentrates in the central location, known as the central bright fringe, the amplitude and intensity distribution is shown in Figure 2.1 and 2.2:

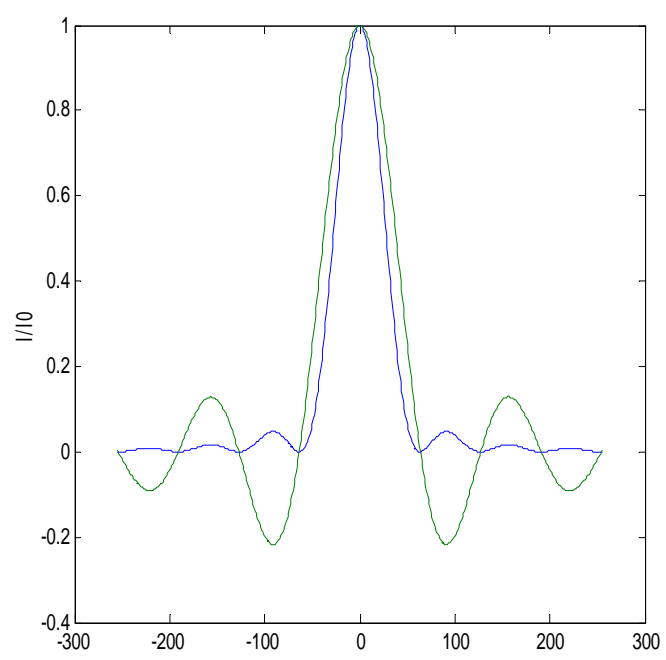

Figure 2.1. Light intensity and amplitude distribution of the diffraction fringes. 

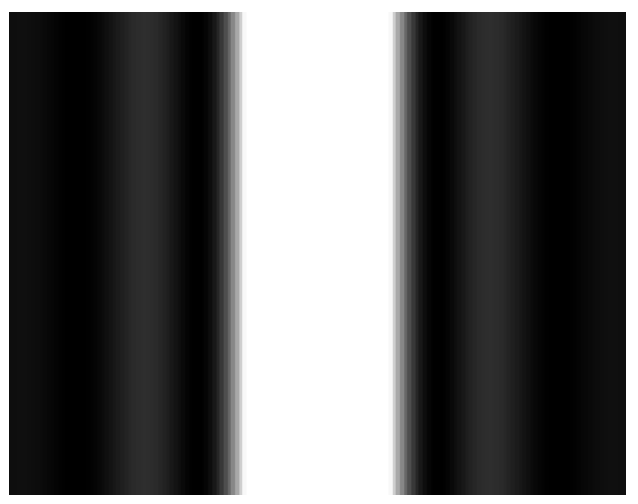

Figure 2.2. Simulation of the diffraction pattern.

According to the formula (2.1), the dark stripes spaced in the diffraction pattern equidistantly, when $\beta=0, \pm \pi \cdots \pm n \pi$. Dark streaks distribution is effected by the slit width the system complete the diameter measurement by extracting dark stripes position accurately from the diffraction pattern, Formula (2.2) deformation is:

$$
\left(\frac{\pi b}{\lambda}\right) \sin \theta=k \pi
$$

Using far field condition on the expression (2.3), we get:

$$
b=\frac{k L \lambda}{x_{k}}
$$

where $x_{k}$ for the distance, between the central bright fringe and the class $\mathrm{k}$ dark stripes, $L$ for the viewing screen and the slit distance, $\lambda$ for wavelength of the measuring light.

Usually it takes CCD device as the sensor for diffraction pattern, since the main power of diffraction pattern focused on the central bright fringe, thus cause the saturation on the corresponding position of the CCD detector, thereby produced adverse effects on the precise determination of the location of the central bright stripes. Expansion the formula (2.4) step by step we get:

$$
x_{k}=\frac{k L \lambda}{b}, x_{k-1}=\frac{(k-1) L \lambda}{b} \ldots x_{k-n}=\frac{(k-n) L \lambda}{b}
$$

And we can further get:

$$
\Delta x=x_{k}-x_{k-1}=\frac{L \lambda}{b}
$$

\section{Dark Points Extraction Algorithm Based on Weighted Thought}

As mentioned before, in the process of the measurement, the determination accuracy of the dark stripe position will directly affect the accuracy of the subsequent calculations. The algorithm used in the traditional applications is relatively simple, by using a point by point comparison, determines the position of the minimum value in the diffraction pattern, The formula is as follows:

$$
\left\{\begin{array}{l}
X_{i+1}>X_{i} \\
X_{i-1}>X_{i} \\
X_{i}<q \bar{X}
\end{array}\right.
$$

where the $\bar{X}$ is the average value of the stripes, $\mathrm{q}$ is a coefficient, usually the value is less than 1 , the specific value is selected based on the actual system. When the light intensity of certain point met with the restrictions will be determined as a minimum point in the diffraction fringes. The algorithm could be satisfactorily complete the extraction of the position of dark fringes in an ideal environment. Actual measurement process, however, the noise will be the inevitable result of a impact of the diffraction patterns. As shown in Figure 3.1, it is the actual measured diffraction fringes signals and the results obtained after the digital filtering:

However, when faced with the actual system noise, the algorithm's performance is not that satisfactory, since the filter can not completely filter out noise. It is due to the single comparison range of the algorithm, and the signal's distortion, which caused by the noise, is randomly distributed in the diffraction fringes. Therefore, When facing a partly signal distortion in that case, the algorithm generates the misjudgment of the diffraction fringes and which will further coarse the extraction error.

This paper presents a dark stripe extraction algorithm, by taking a multi-range comparison and then carry on a weight calculated according to the comparison result, this algorithm can suppress the error which arising in the extraction process. Its specific calculation is as follows:
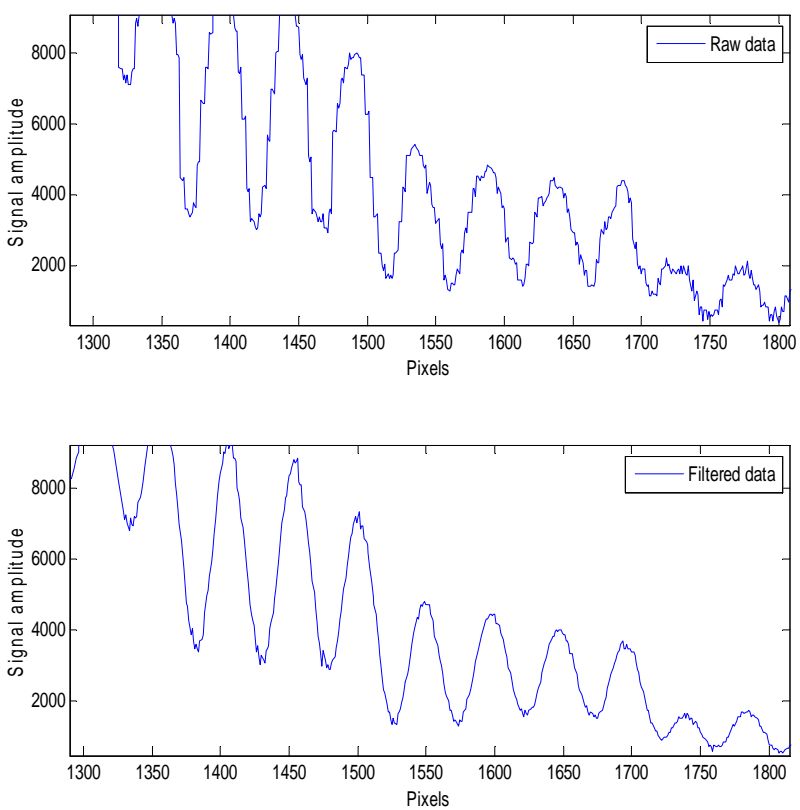

Figure 3.1. The original data and the filtered data. 


$$
\begin{aligned}
& M_{k}=\left\{\begin{array}{l}
X_{i+k}>X_{i} \\
X_{i-k}>X_{i}
\end{array} \ldots \ldots(k=1,2,5)\right. \\
& M=\sum M_{K} \ldots \ldots(k=1,2,5)
\end{aligned}
$$

$M_{k}$ is a boolean variable, when the expression in parentheses is true the $M_{k}$ equal to 1,And otherwise it's 0 . Weighted value of $M_{k}$ and cumulate it to get value $M$. By doing threshold decision on $M$ value we can get a final judgment results. Using the computer software to simulate the algorithm obtained the following results as showed in Figure 3.2:

From the simulation figure it can be seen, that calculated by comparison of multi-range for diffraction fringes can overcome the deficiencies of the traditional extraction algorithm. The reason is that the improved extraction algorithm increased the restrictions of the extraction process to reduce the misjudgment of the minimum point compared to traditional extraction algorithm.

\section{Curve Fitting Algorithm Based on Least Squares Principle}

The improved dark stripe extraction algorithm only completes exclusion of gross error. In order to enhance the measurement accuracy of the system, this paper take use of the least squares curve fitting of the diffraction fringes data, thus completing the sub-pixel subdivision of dark stripes' location. According to the former analysis of the diffraction fringe signal, it shows that the signal curve of the dark stripe is similar to the parabolic curve. So the curve equation is assumed to be:
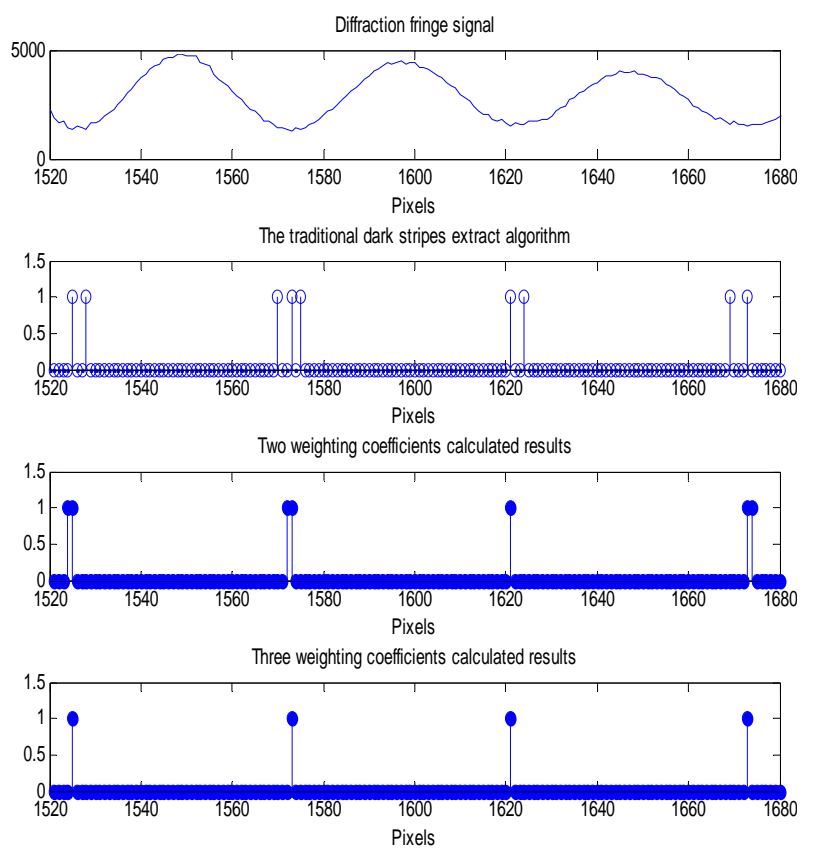

Figure 3.2. The dark stripes extraction algorithm simulation.

$$
f(x)=a_{0}+a_{1} x+a_{2} x^{2}
$$

According to the least squares principle:

$$
F\left(a_{0} a_{1} a_{2}\right)=\sum_{i=1}^{n}\left(I\left(x_{i}\right)-f\left(x_{i}\right)\right)^{2}
$$

where $I(*)$ is the actual measurement obtained measured values, partial derivative of the expression(4.2), we get:

$$
\frac{\partial F\left(a_{0}, a_{1}, a_{2}\right)}{\partial a_{i}}=0 \ldots \ldots(i=0,1,2)
$$

Thereby obtain the equation:

$$
\left(\begin{array}{ccc}
n & \sum x_{i} & \sum x_{i}^{2} \\
\sum x_{i} & \sum x_{i}^{2} & \sum x_{i}^{3} \\
\sum x_{i}^{2} & \sum x_{i}^{3} & \sum x_{i}^{4}
\end{array}\right)\left(\begin{array}{l}
a_{0} \\
a_{1} \\
a_{2}
\end{array}\right)=\left(\begin{array}{c}
\sum I\left(x_{i}\right) \\
\sum x_{i} \bullet I\left(x_{i}\right) \\
\sum x_{i}^{2} \bullet I\left(x_{i}\right)
\end{array}\right)
$$

In this expression, the $\sum$ behalf the cumulative for all sampling points, by solving the above equation (4.4), we can further obtain the expression:

$$
\frac{a_{1}}{a_{2}}=\frac{P\left[n \sum x_{i}^{4}-\left(\sum x_{i}^{2}\right)^{2}\right]-Q\left[n \sum x_{i}^{3}-\sum x_{i} \sum x_{i}^{2}\right]}{Q\left[n \sum x_{i}^{2}-\left(\sum x_{i}\right)^{2}\right]-P\left[n \sum x_{i}^{3}-\sum x_{i} \sum x_{i}^{2}\right]}
$$

where:

$$
\begin{aligned}
& P=\left[n \sum x_{i} I\left(x_{i}\right)-\sum I\left(x_{i}\right) \sum x_{i}\right] \\
& Q=\left[n \sum x_{i}^{2} I\left(x_{i}\right)-\sum I\left(x_{i}\right) \sum x_{i}^{2}\right]
\end{aligned}
$$

The dark stripe of diffraction fringes is located on the minimum point of the curve, and therefore the derivation of the obtained equation(4.7):

$$
\frac{d f(x)}{d x}=0
$$

with this expression we can deduce the location of the dark points:

$$
x_{0}=-\frac{a_{1}}{2 a_{2}}
$$

Take formula (4.5) into the above expression it can obtain the position of the dark points. Carry on the fitting calculation by the computer on the calculated diffraction fringes location above, we get the simulation diagram as Figure 4.1:

To complete the calculation of subdivision of the CCD pixel. By fitting the data arround each dark point of the overall diffraction fringe pattern, we can obtain the accurate position of the dark points, and thus the measured diameter could be calculated according to the position of the dark points.

\section{Experimental Data and Analysis}

For the filaments whose nominal diameter is $100 \mathrm{um}$, 


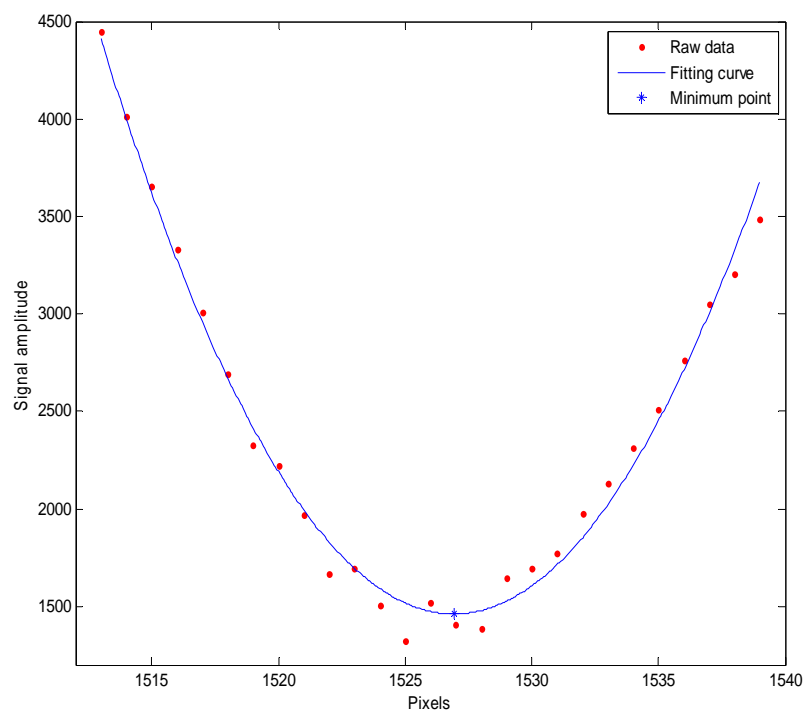

Figure 4.1. Least-squares fitting algorithm simulation.

Table 5.1. Sub-pixel segmentation results.

\begin{tabular}{ccccc}
\hline & 1 & 2 & 3 & 4 \\
\hline$\Delta x$ & 45.39 & 44.29 & 44.56 & 44.64 \\
$\mathrm{~B}(\mathrm{~mm})$ & 0.0982 & 0.1044 & 0.1027 & 0.1011 \\
& 5 & 6 & 7 & 8 \\
$\Delta x$ & 45.16 & 45.48 & 44.62 & 46.61 \\
$\mathrm{~B}(\mathrm{~mm})$ & 0.0996 & 0.0977 & 0.1008 & 0.0926 \\
\hline
\end{tabular}

calculate by the algorithm that presented in this paper, we obtain the following data that showed in the Table 5.1 according to the formula (2.6):

The mean square error is:

$$
\sigma=\frac{1}{n} \sum_{i=1}^{n}\left(x_{i}-b\right)^{2}=0.0034
$$

Deformed and take the differential to the formula(2.6) we get:

$$
d b=\frac{L}{\Delta x} d \lambda+\frac{\lambda}{\Delta x} d L+\left|\frac{\lambda L}{\Delta x^{2}} d \Delta x\right|
$$

In this expression the first term is the error introduced by the laser linewidth. The second one is error of the imaging lens' focal length, can be regarded as a system error, and it can be eliminated by calibration the system; the third term is the error of the measurement process itself. And we can infer that when the object larger the diameter, the smaller the pitch of dark diffraction fringes, which leading to a larger measurement error.

\section{Conclusions}

This paper summarizes the traditional dark stripes extraction algorithm, based on which proposed a improved dark fringe extraction algorithm based on the weighted thinking and used the least squares fitting algorithm to subdivide the location of the dark spots. According to the simulation results, the feasibility of the algorithm is verified. Finally, by using the experimental data we analyzed the measurement error of the system, and it can be tell that the improved dark stripes extraction algorithm achieved better results.

\section{REFERENCES}

[1] A. K. Soraya, "Measurement of Wire Diameter by Optical Diffraction," Optics\&Laser Technology, Vol. 36, 2004, pp. 63-67.doi:10.1016/S0030-3992(03)00134-8

[2] F.S. Zhang and C. Liu, "Superpresicion Laser Diffration Measuring System for Fine Diameter,"Chinese Journal of Scientific Instrument, Vol. 22, 2001, pp.149-150.

[3] L. Q. He, Y. Gao and G. G. Wang, "Calibration Method for CCD Laser Diffration Filament Diameter Measurement System," Journal of Jilin University, Vol. 38, 2008, pp.182-184.

[4] Y. Zhang, "Two Improving Methods of Object Orientation Precision by CCDs," Journal of Optoelectronics.Laser, 2003.

[5] D. Y. Sun, G. X. Zhou and D. Liu, "Measurement of Filament by Diffraction Method," Instrumentation Technology, 2002.

[6] K. Wang and Z. J. Mao, "Actualing Least-Squares Curve-Fitting Based on Matlab," Journal of Beijing Broadcasting Instute, Vol. 12, pp. 52-56.

[7] K. L. Ding, Y. Z. Shen and J. K. Ou, "Methods of Line-Fitting Based on Total Least-Squares," Journal of Liaoning Technical University, Vol. 29, 2010, pp.44-47. 